\title{
RAPID ANALYSIS METHOD FOR HIGH FREQUENCY SIGNAL TIME DOMAIN CHARACTERISTICS IN ON-LINE MONITORING OF ELECTRICAL EQUIPMENT
}

\author{
Qilong Wang ${ }^{1}$, Lingling Song ${ }^{1}$ \\ ${ }^{1}$ Department of Electrical and Electronic Engineering, Yantai Vocational College, \\ Yantai, Shandong 264670, China. \\ Email:wql_wangqilongyt@126.com
}

\begin{abstract}
In order to solve a series of problems existing in the monitoring of electrical equipment, such as long-time interval of data collection and poor analysis performance, in this research, a rapid analysis method of high frequency signal time domain characteristics is designed using high frequency signal characteristics analysis algorithm. Firstly, the structure of high-frequency signal characteristics analysis algorithm is designed; the method of signal time domain characteristics extraction is introduced; the extraction method of high frequency signals time domain characteristics is optimized to obtain the signal rapid analysis method. Then, the signal characteristic structure is analysed. Finally, Mathlab simulation software is used to analyse the performance of the rapid analysis algorithm of high frequency signal characteristics. The results show that the high frequency signal characteristics analysis algorithm designed in this research includes waveform time domain characteristics; the data files that need to be stored are obtained by the statistical method of characteristic signals; the accuracy, reliability, and rapidity of the proposed algorithm are verified by simulation experiments. This research can promote the development of intelligent electrical equipment monitoring systems.
\end{abstract}

Keywords: Electrical Equipment; Time Domain Characteristics; High Frequency Signal; On-Line Monitoring; Rapid Analysis Method.

\section{Introduction}

With the development of society, people's production and life are inseparable from the electric power system, which plays a very important role in daily life. With the further development of industrialization, higher and higher requirements are placed on the power system.

The increase of voltage and capacity are the demands of electrical equipment (such as large motors and transformers) [1].

Once the electric power equipment is not stopped according to the plan, it will cause huge economic loss and great social impact to industrial production. Therefore, the normal operation of electric power equipment is of great significance to the stable development of the whole society.

According to the relevant operation data, the current faults of electrical equipment mainly come from the insulation fault, therefore, mastering the insulation health of electrical insulation equipment is one of the important factors to solve the fault of electrical equipment. With the development of science and technology, on-line monitoring system is proposed. On-line monitoring refers to the continuous partial discharge measurement of electrical equipment during normal operation; the monitoring results are regularly uploaded to obtain real-time information about the health of the electrical equipment; compared with offline monitoring, which requires a long time to wait for power failure and has errors between the measurement environment and the real fault, online monitoring can be used to monitor and diagnose the faults of electrical equipment to realize intelligent maintenance; online monitoring has the characteristics of sensitive response and high monitoring accuracy $[2,3]$.

In the process of online monitoring of electrical equipment, the sensor will collect high-frequency signals, which contain a large amount of partial discharge information, and the research on the signal characteristics of partial discharge information is the main research object to solve the fault of electrical equipment. At present, there are many methods to measure partial discharge [4].

First, when partial discharge occurs, a pulse current signal is generated; by detecting the pulse current signal, information about partial discharge can be obtained. Secondly, according to the non- 
electric quantity information generated in the partial discharge process, the corresponding non-electric quantity information monitoring method appears.

For example, there are chemical monitoring method, light measurement method, ultrasonic monitoring method, and infrared thermal imaging method for smell, light, sound, heat, and other information. Finally, with the rapid development of sensor technology, the signals obtained by online partial discharge monitoring are widely distributed in the frequency band, including the conventional low-frequency pulse current method, broadband pulse current method, ultrasonic monitoring method, and ultra-high frequency monitoring method [5,6].

Even at low frequencies, the upper cut-off frequency can be as high as MHz. Therefore, for the monitoring of electrical equipment, during the operation of the equipment, the insulation system will be affected by the environment around the electrical equipment, heat, mechanical and other factors. These factors have a certain coupling relationship, and the traditional monitoring system seldom pays attention to these cases. In addition, with the fluctuation of the power system and the change of the health state of the electrical equipment group, the operation state of the electrical equipment is constantly changing [7].

Therefore, it is an urgent problem to study the rapid analysis method of on-line monitoring of electrical equipment.

To sum up, in order to improve the long-time interval and poor analysis performance of traditional high-frequency signal monitoring and data collection, in this research, a rapid analysis method of high frequency signal characteristics in time domain in on-line monitoring of electrical equipment is designed based on the rapid analysis algorithm of high frequency signal characteristics.
Firstly, the structure of high-frequency signal characteristics analysis algorithm is designed; the extraction method of signal time domain characteristics is introduced; the signal rapid analysis method is optimized.

Then the optimization results of the signal characteristic structure are analysed. Finally, the performance of the high frequency signal characteristics analysis algorithm is simulated. It is expected to provide a good research idea for the online monitoring of electrical equipment.

\section{Methods}

\subsection{High frequency signal characteristics analysis algorithm structure}

The characteristic analysis algorithm of highfrequency discharge signals can be divided into three parts: extracting the time-domain characteristics of waveform, analysing the extraction results, and judging whether the waveform is stored or not.

Therefore, the algorithm structure must contain these three main parts, as shown in Figure 1.

In the real-time analysis algorithm, extracting waveform time-domain characteristics refers to the process of converting original data into waveform data. By extracting the extremum points in the process of signal fluctuation, the original data is converted into multiple fluctuation characteristics points [8]. Statistical analysis is the process of collecting and classifying fluctuation data to obtain statistical results of current signals. Then the data before the statistical results are compared to get the change.

The result judgment is the process of deciding whether to enter the waveform memory according to the set threshold or other conditions.

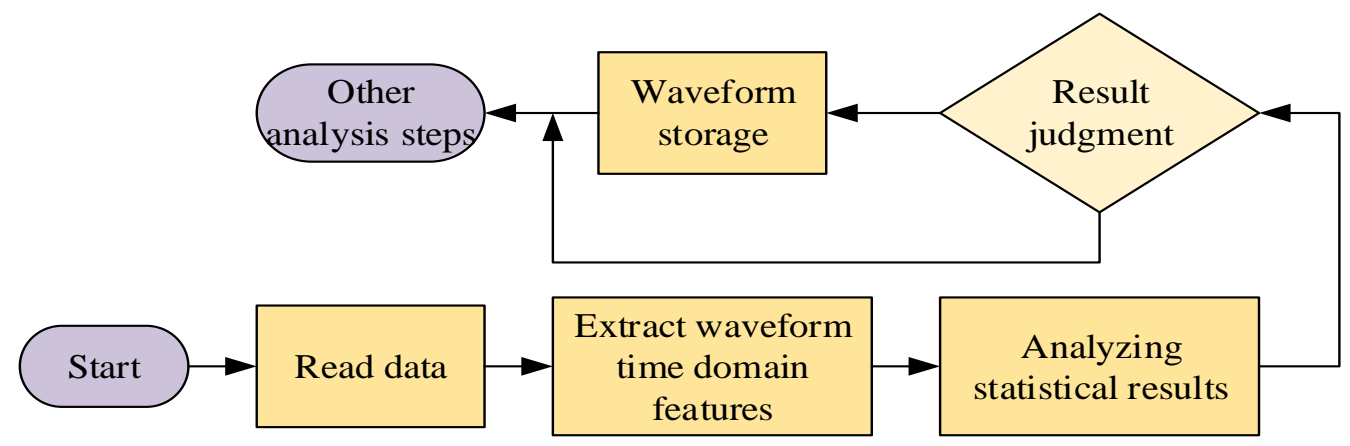

Figure 1: The realization block diagram of real-time analysis.

\subsection{Signal time domain characteristics extraction method}

Since the existing signal analysis methods can't achieve rapid processing of high-frequency signals, therefore, the analysis method of characteristics point extraction is designed in this research. This method is based on the principle of human eye recognition pulse, which is characterized by rapid analysis, low extraction error rate, and comprehensive response of main signal characteristics [9]. 
The human eye recognizes the pulse signal in three steps.

Step 1: general judgement. When the human eye sees the signal, it traverses the entire screen to get the range and magnitude of the signal's fluctuations. If the fluctuations are periodic and similar, they are classified into one category. For a machine, the envelope of the analogue signal can simulate the rough judgment process of the human eye, but the information contained in the envelope is incomplete and usually needs to be used in combination with other means. From the overall distribution of statistical signals, it can be concluded that the obtained statistical data can be combined in some way to simulate the classification steps of human eyes [10].

Step 2: when judging the pulse, the speed and size of the current fluctuation are determined according to the wave of the signal, and the pulse is determined. The human eye will look for signal fluctuations during periods of rapid growth/decline in signal fluctuations. In the process of judging the amplitude, as the pulse oscillation decays, the end point of the pulse is gradually determined. Then the duration of the pulse is obtained, and the comprehensive characteristics of the pulse are obtained. Finally, the pulse fluctuation is evaluated according to wave frequency, fluctuation amplitude, fluctuation time, and oscillation frequency [11].

Step 3: for multiple pulses, the human eye moves one pulse to another and compares other parameters such as contour, amplitude, duration, and position. Finally, by comparing the combined pulse distribution with the knowledge and experience mastered, the evaluation result of pulse change is obtained [12].

Figure 2 is the schematic diagram of the step framework of the characteristic extraction method. For the characteristic extraction method, for the incoming signal, white noise is common in highfrequency signals and has boundary property, therefore, extreme points can be extracted from a group of white noise signals, and the maximum value can be denoted as the reading value of the separation noise, denoted as Thresl. In practical application, due to the different structure and technology of different generator sets, it is necessary to use different analysis methods to determine the threshold of different generator sets. The judgment of the extreme point may happen differently.

Assuming that the last limit point of extraction is the minimum value, in terms of the steps of eigenvalue extraction in the signal, the difference between data needs to be calculated first. If the change direction compared is the same, the point is given up. When the change direction is different, it is compared with the pre-set condition. If the change direction is greater than the pre-set point, the current point is overridden; if the default condition is the same, the new characteristics point is recorded; if less than the default condition, the point is discarded.

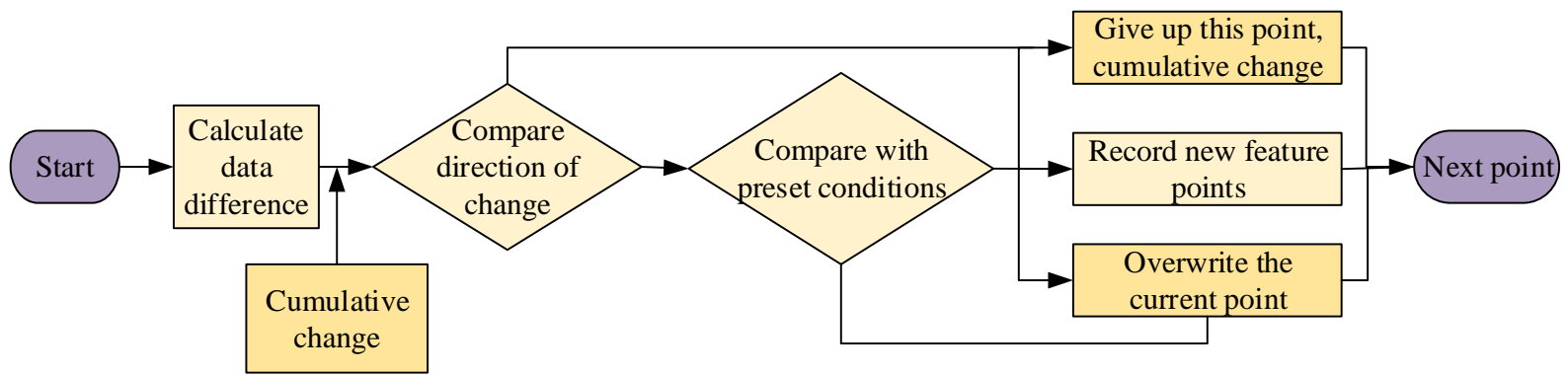

Figure 2: The block diagram of characteristics extraction method.

The influence of noise can't be completely removed using only the wave threshold method for the characteristics noise separation. Because the characteristic points at the beginning and end of the pulse become inaccurate. Therefore, the noise signal needs to be removed by the method of slope threshold.

The definition expression of slope is as follows:

$$
\begin{aligned}
& \operatorname{slope}(k)=\frac{d(k)-d(k-1)}{n(k)-n(k-1)} \\
& |\operatorname{slope}(k)|<\text { Thres } 2
\end{aligned}
$$

In Eq. 1 and Eq. 2, k represents the serial number of the current eigenvalue; Thres2 represents the appropriate threshold.
When its threshold value meets Eq. 2, it means that the current characteristics point belongs to the starting point of the above mixed signal noise, which can be used to process the fluctuation segment, and the data of the characteristics points before and after correction is required.

In the process of de-noising, the fluctuations of different pulses can be separated according to the location of noise, so the original files can be divided into multiple wave groups. Since the end of the pulse usually oscillates less, the end of the segment is not modified, and the beginning of the wave segment is modified to an adjacent extreme. If the corrected change is less than Thresl, it means no correction is required. 
The first characteristic point is taken as the starting point of the interval. Finally, parameters of relevant characteristics points are updated in turn to obtain high-quality characteristics quantities [13].

In the extraction process, if the wave has a small number of oscillations, it is considered that the wave does not have pulse characteristics according to the above pulse characteristics, so the characteristics points are deleted. The fluctuation frequency threshold is set as Thres3. If the fluctuation frequency in a set of fluctuations is less than the threshold, the value is set to 3 . The judgment equation is as follows.

$$
n . e n d(k)-n . s t a r t(k)<\text { Thres } 3
$$

In Eq. 3, $\mathrm{k}$ represents the serial number of the current eigenvalue; end represents the end point of the wave; start represents the starting point of the wave; $\mathrm{n}$ represents the position number in the characteristics sequence table.

To sum up, the schematic diagram of the judgment process of characteristics SNR separation is shown in Figure 3.

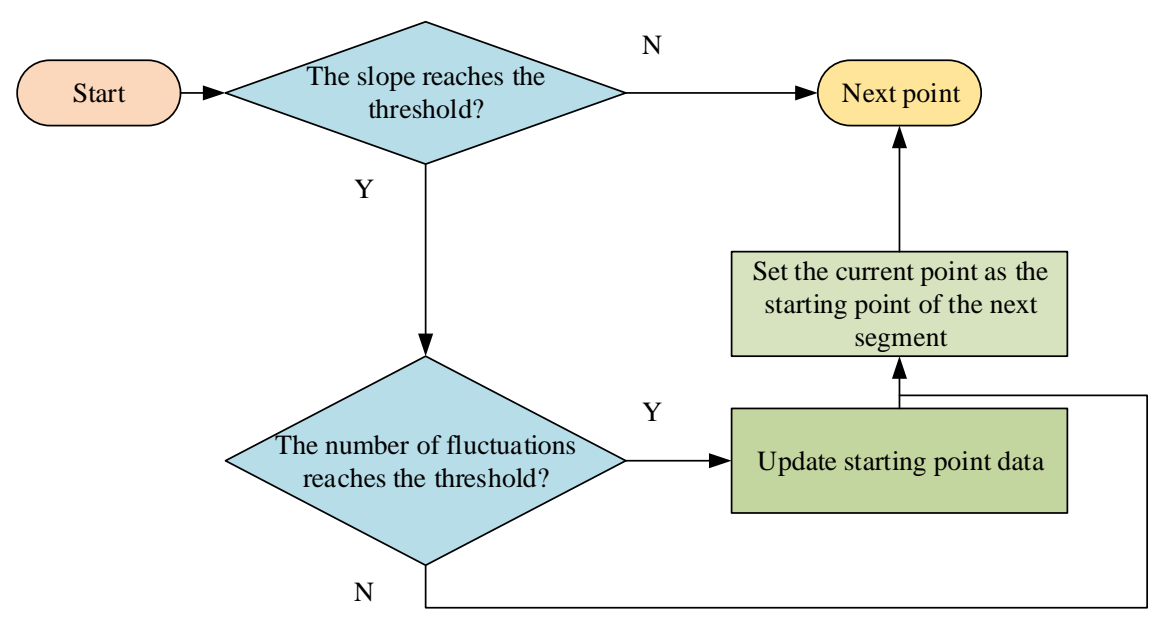

Figure 3: Flow chart of characteristic signal-noise separation judgment.

In the time domain of high frequency signal, there are four characteristic quantities: maximum amplitude of wave, frequency of wave oscillation, duration of wave, and equivalent velocity of wave oscillation.

The maximum fluctuation range refers to the maximum value in a group of fluctuations and can be denoted as Ap.

The frequency of fluctuation and oscillation refers to the sum of the number of characteristic points between the starting point of the fluctuation interval and the end point of the fluctuation interval, which is also the total number of peaks and valleys between the region. It is expressed as Eq. 4.

$$
n p v(k)=n . e n d(k)-n . \operatorname{start}(k)
$$

Duration of fluctuation refers to the time difference between the end point of fluctuation segment and the beginning of fluctuation, which can be expressed as Eq. 5.

$$
t(k)=\text { time.end }(k) \text {-time.start }(k)
$$

The equivalent oscillation velocity refers to calculating the average oscillation velocity within a group of fluctuations. The first oscillating process is the time that two oscillating processes go through.

By calculating all oscillation processes, inverse reciprocal of the average value of oscillation time is obtained to get the equivalent fluctuation frequency, which is expressed as Eq. 6.

$$
f_{e}=\frac{n}{\sum_{i=1}^{n} t_{i}}
$$

\subsection{Optimization of signal rapid analysis methods}

In order to improve the operating efficiency of the rapid analysis method for high-frequency signals of electrical equipment, in addition to the rational design of the analysis method, it is also necessary to reconstruct the analysis method according to the actual situation of the application of the analysis method [14].

The measures taken to reduce the running time of analysis methods in this research are to select the right runtime environment and programming language, which prevents the runtime of the analysis method from being hampered by other factors.

Since this analysis requires raw data from the hardware and involves the bottom of the system, it is implemented in $\mathrm{C}++$ to ensure that the run time increases with the increase in data processing power.

Therefore, it is necessary to reasonably combine extraction methods to reduce the number of operations and the time consumed by characteristics extraction methods. In order to reduce the amount of operation data, only the number of characteristics 
points and fluctuation range are recorded during the execution. Once extracted, the displayed data is retrieved. After testing, it is basically possible to extract 50,000-100,000 characteristics points from a single original file, which greatly reduces the amount of data. Therefore, the analysis step takes less time and can be completed in $10 \mathrm{~ms}$.

The characteristic signal-noise separation is decomposed into three periods. The first loop is completed by traversing the characteristics point store table to calculate the slope. Then it fluctuates piecewise to determine the starting and ending points of the fluctuation segments, clean up the nonconforming fluctuations, and complete the second cycle.

Finally, the parameters are calculated, the statistics is completed, and the third cycle is finished.

\section{Results and Discussion \\ 3.1 Analysis of signal characteristics}

Based on the analysis of historical signals, the signals collected in different short periods of time have the following characteristics: firstly, the change of signal is relatively slow, and there are few instantaneous mutations; secondly, in general, the instantaneous change is related to the special situation. Due to the complex on-site environment of electrical equipment, the fluctuation range can be determined based on historical data. When the number of normal wave pulses remains unchanged, the amplitude changes. In order to improve the quality of stored data, the judgment strategy needs to be distinguished by fluctuations and pulse changes.

Figure 4 is the pulse amplitude change process diagram. Under the condition that the number of fluctuations extracted from the corresponding pulse remains unchanged, the fluctuation arrangement will increase or decrease significantly.

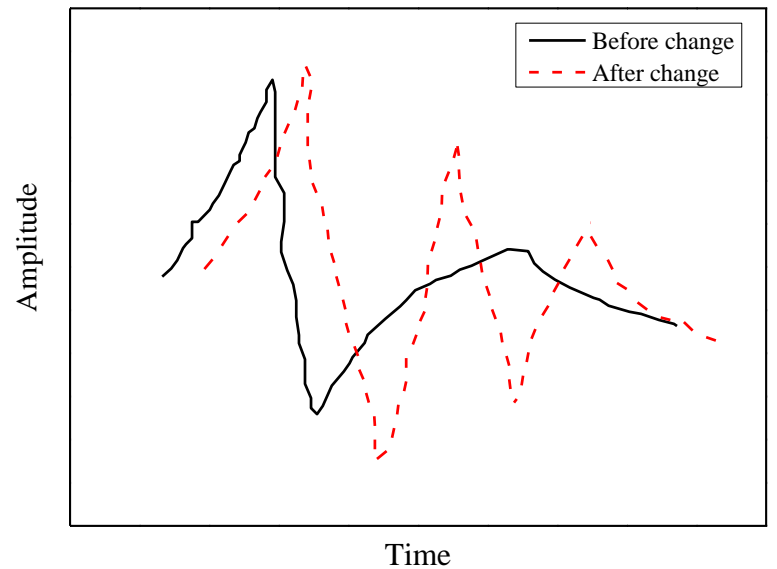

Figure 4: The pulse amplitude variation process diagram.
Figure 5 is the distribution of characteristics points, and Figure 6 is local magnification of characteristics point distribution. The data of the characteristic points will first increase and then decrease as the amplitude increases.

The reason for the cliff-like drop is that when the amplitude reaches the maximum fluctuation value of a class of pulses, such pulses will disappear in the subsequent data.

Thus the variations of different pulses can be counted by classifying the fluctuations according to the amplitude, and the raw data can be divided into three regions according to the magnitude of the fluctuations.

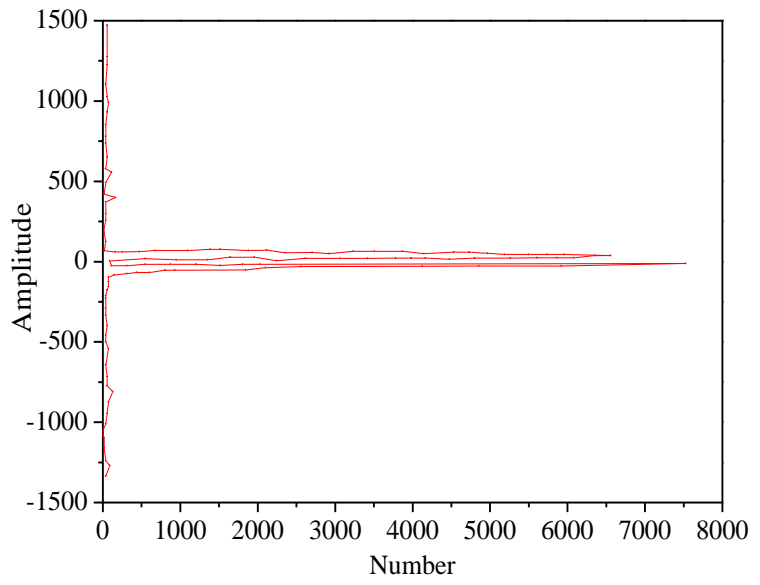

Figure 5: Characteristics points distribution.

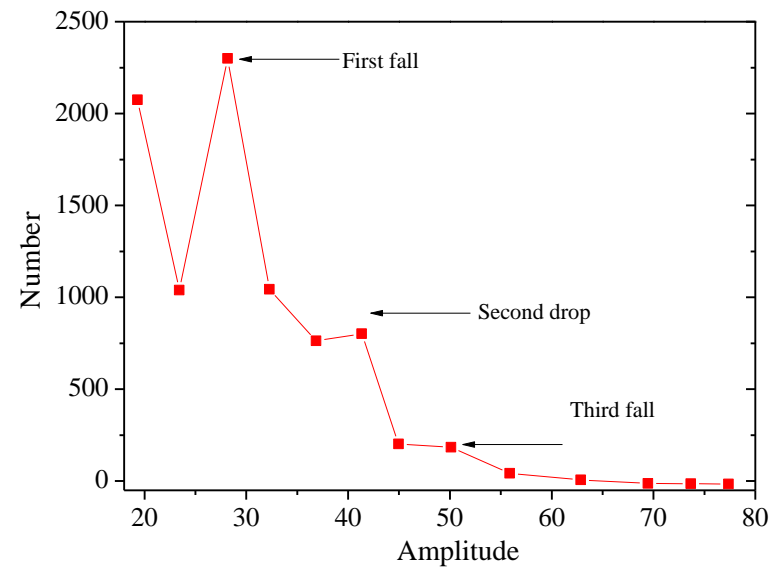

Figure 6: Local magnification of characteristics point distribution.

According to the real-time analysis results of data characteristics points, the fluctuation of data characteristics points can be divided into three levels: high level, middle level, and low level. In general, the wave head has a large fluctuation range, which reflects the pulse amplitude.

The components with moderate fluctuation amplitude reflect the pulse coupling and oscillation process. 
The components with lower fluctuation amplitude can reflect the attenuation process of the wave tail.

According to the time-domain characteristics of the pulse, the high, medium, and low regions can be subdivided. Each type of area can be further divided into three panes based on the size of the amplitude.

The maximum fluctuation of the signal is calculated again. The maximum pane is set to the exception detection pane. This pane is usually numbered 0 .

Because of the structure between the units, the sensor signals are tracked to different degrees, so it is necessary to divide the panes according to the characteristics of the unit data files.

Once the panes are identified, the partition criteria should not be changed in real time to ensure comparability of the data files, but fluctuations within the corresponding intervals can be calculated.

There are 10 Windows in one direction, and the fluctuation range is positive and negative respectively.

Thus, there are 20 statistics in one file, plus the maximum of the positive and negative fluctuations, for a total of 42 .

Files with small to large margins on the positive side of each file are called layer 1-10 panes, and files with large margins on the negative side are called layer 11-20 panes.

\subsection{Performance analysis of high frequency signal characteristics analysis algorithm}

In order to verify the performance of high frequency signal characteristics analysis algorithm, the Mathlab software is adopted for simulation experiment.

In this research, the single exponential attenuation oscillation function and double exponential attenuation oscillation function are used for the simulation signal source, and their expressions are shown in Eq. 7 and Eq. 8.

It can be used for random oscillation pulse and silicon-controlled pulse.

$$
\begin{aligned}
& s_{1}(t)=A_{1} e^{-t / \tau} \sin \left(2 \pi f_{c} t\right) \\
& s_{2}(t)=A_{2}\left(e^{-1.5 t / \tau}-e^{-2.8 t / \tau}\right) \sin \left(2 \pi f_{c} t\right)
\end{aligned}
$$

In Eq. 7 and Eq. 8, A represents the pulse amplitude, $\tau$ denotes the duration of the pulse; $f_{c}$ represents the dominant frequency of pulse fluctuation.

Simulation parameters and superimposed noise of different simulation pulses are shown in Table 1.

Among them, all pulse transmission interval is $2 \mathrm{~ms}$, and each simulation waveform file protects 50 identical pulse waves.

Since the noise model is relatively obvious, the signal polluted by noise in signal simulation can be expressed as Eq. 9.

$$
f(t)=s(t)+\alpha \cdot \sin (\omega \mathrm{t})+n(t)
$$

The random oscillation pulse test results are verified by extracting the effect diagram of the simulation signal and the effect diagram of the four extracted parameters.

Figure 7 shows the test results of random oscillation pulse. Table 2 shows the extraction results of random oscillation pulse parameters.

It can be concluded from figure 7 and table 2 that the extraction effect uses half of the measured peakto-peak value.

Therefore, the high frequency signal time domain characteristics extraction results have a high accuracy rate, which is consistent with the expected effect.

Table 1. Simulation pulse parameters and different simulation pulse stack noise.

\begin{tabular}{|l|c|c|c|c|l|l|}
\hline $\begin{array}{l}\text { Pulse } \\
\text { type }\end{array}$ & $\begin{array}{l}\text { Simulation } \\
\text { expression }\end{array}$ & $\begin{array}{l}\text { Amplitude } \\
\mathbf{A} / \mathbf{m V}\end{array}$ & $\begin{array}{l}\text { Attenuation } \\
\text { coefficient } \\
\tau / \mathbf{u s}\end{array}$ & $\begin{array}{l}\text { Oscillation } \\
\text { frequency } \\
f_{c} / \mathbf{M H z}\end{array}$ & $\begin{array}{l}\text { Superimposed } \\
\text { noise type }\end{array}$ & $\begin{array}{l}\text { Noise } \\
\text { parameters }\end{array}$ \\
\hline $\begin{array}{l}\text { Random } \\
\text { oscillation } \\
\text { pulse }\end{array}$ & Eq. 7 & 120 & 2.2 & 4.9 & White noise & $\mathrm{N}(0,4)$ \\
\hline $\begin{array}{l}\text { Silicon } \\
\text { controlled } \\
\text { pulse }\end{array}$ & Eq. 8 & 450 & 8.1 & 0.7 & $\begin{array}{l}\text { Narrowband } \\
\text { disturbance }\end{array}$ & $\begin{array}{l}\text { Frequency: } \\
\text { amplitude: } \\
\text { amplitud } \\
10 \mathrm{mV}\end{array}$ \\
\hline
\end{tabular}




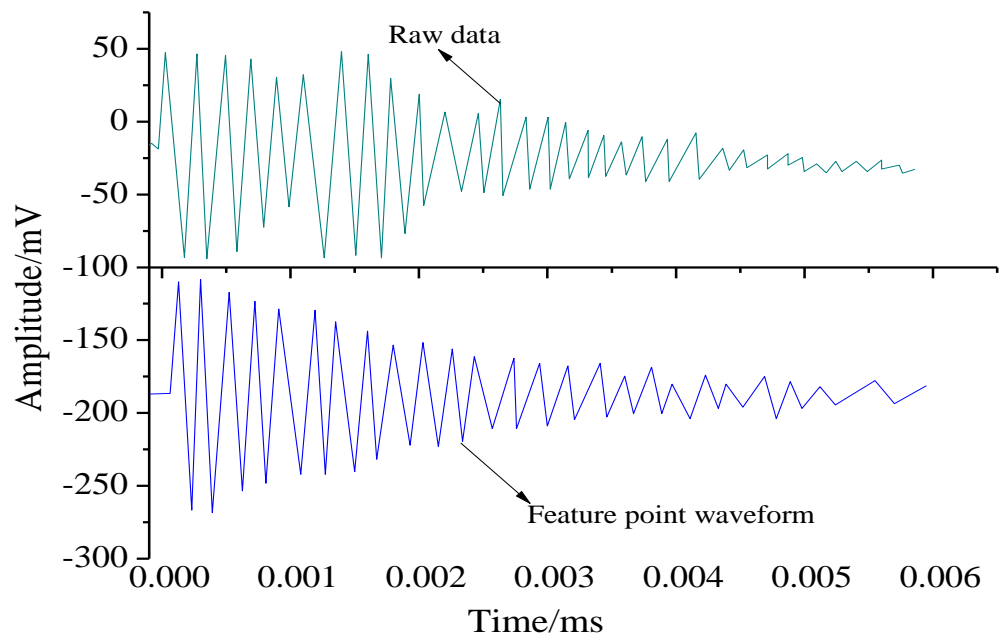

Figure 7: Effect diagram of random oscillation pulse simulation signal extraction.

Table 2. Random oscillation pulse parameter extraction results

\begin{tabular}{|l|c|c|c|}
\hline Parameter type & Measured values & Accurate values & Accuracy rate/\% \\
\hline Amplitude & $87.53 \mathrm{mV}$ & $87.1 \mathrm{mV}$ & 99.4 \\
\hline Oscillation speed & $4.72 \mathrm{MHz}$ & $4.8 \mathrm{MHz}$ & 98.3 \\
\hline Number & 54 & 51 & 94.4 \\
\hline $\begin{array}{l}\text { Duration of } \\
\text { oscillation }\end{array}$ & $5.3 \mathrm{us}$ & $5.7 \mathrm{us}$ & 92.9 \\
\hline
\end{tabular}

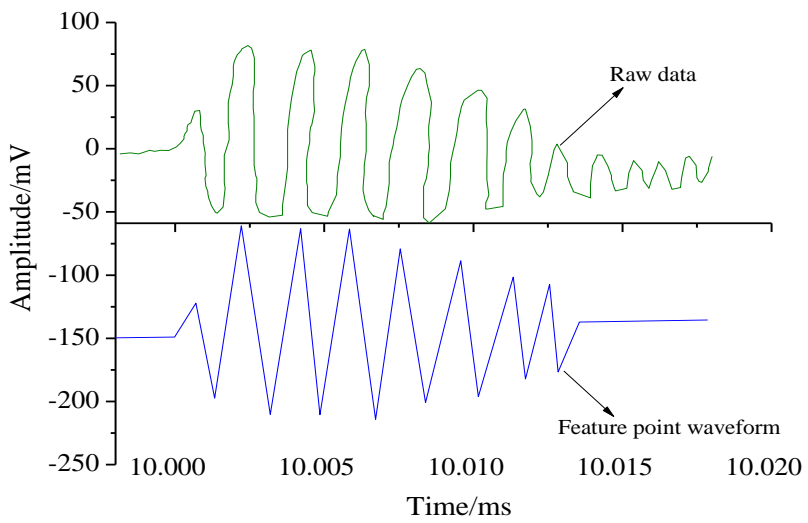

Figure 8: The effect diagram of SCR pulse simulation signal extraction.

Table 3. The extraction results of SCR pulse parameters

\begin{tabular}{|c|c|c|c|}
\hline Parameter type & Measured values & Accurate values & Accuracy rate/\% \\
\hline Amplitude & $86.4 \mathrm{mV}$ & $86.1 \mathrm{mV}$ & 99.6 \\
\hline Oscillation speed & $0.72 \mathrm{MHz}$ & $0.70 \mathrm{MHz}$ & 97.2 \\
\hline Number & 20 & 18 & 90.0 \\
\hline Duration of oscillation & $16 \mathrm{us}$ & $15.6 \mathrm{us}$ & 97.5 \\
\hline & & & \\
\hline
\end{tabular}

The silicon-controlled rectifier (SCR) pulse test results are verified using the same signal source as the random oscillation pulse test results. The renderings extracted from the simulation signal and the four extracted parameters ae verified. Figure 8 shows the effect diagram of SCR pulse simulation signal extraction. Table 3 shows the extraction results of SCR pulse parameters. It can be concluded from Figure 8 and Table 3 that, in the waveform characteristics extraction results, the extraction effect of pulse amplitude and equivalent oscillation velocity is better. In the extraction process, in order to filter out the noise, some information at the end of the pulse is lost, but the overall effect is acceptable.

According to the test results of the two models, the recognition rate of the method proposed in this research is more than $90 \%$, and the difference in 
recognition accuracy is less than $1 \%$. Therefore, the rapid analysis method of high frequency signal in time domain is efficient and reliable.

\section{Conclusions}

In order to solve the problems of slow signal response, multiple interruption of monitoring effect, and low accuracy in the monitoring process of electrical equipment, in this research, a rapid analysis method of high frequency signal time domain is designed. First of all, the structure of high-frequency signal characteristics analysis algorithm and the signal time-domain characteristics extraction method are designed, and the method of signal rapid analysis is optimized. Then the signal characteristic results are analysed. Finally, the performance of the high frequency signal characteristics analysis algorithm is analysed.

The data files to be stored are obtained by analysing the signal characteristics. Simulation software is used to verify the accuracy and rapidity of the high frequency signal characteristics analysis algorithm.

The research provides a good theoretical basis for the rapid analysis of high-frequency signal timedomain characteristics in electrical equipment monitoring. However, there are still some limitations in this research, and the accuracy of the algorithm needs to be further improved.

\section{References}

[1] M. Siegel, M. Beltle, S. Tenbohlen, et al. "Application of UHF sensors for PD measurement at power transformers." IEEE Transactions on dielectrics and Electrical Insulation, vol.24, no.1, pp. 331-339, 2017.

[2] R. U. Maheswari, R. Umamaheswari. "Trends in non-stationary signal processing techniques applied to vibration analysis of wind turbine drive train-A contemporary survey." Mechanical Systems and Signal Processing, vol.85, pp. 296311, 2017.

[3] A. Riccobono, M. Cupelli, A. Monti, et al. "Stability of shipboard dc power distribution: Online impedance-based systems methods." IEEE Electrification Magazine, vol.5, no.3, pp. 55-67, 2017.

[4] X. Wu, L. Han, Z. Wang, et al. "A nonintrusive fast residential load identification algorithm based on frequency-domain template filtering." IEEJ Transactions on Electrical and Electronic Engineering, vol.12, pp. S125-S133, 2017.
[5] C. Mishra, A. K. Samantaray, G. Chakraborty. "Rolling element bearing fault diagnosis under slow speed operation using wavelet de-noising." Measurement, vol.103, pp. 77-86, 2017.

[6] X. Wang, S. Guan, L. Hua, et al. "Classification of spot-welded joint strength using ultrasonic signal time-frequency characteristics and PSOSVM method." Ultrasonics, vol.91, pp. 161-169, 2019.

[7] A.V. Filippov, V. E. Rubtsov, S. Y. Tarasov, et al. "Detecting transition to chatter mode in peakless tool turning by monitoring vibration and acoustic emission signals." The International Journal of Advanced Manufacturing Technology, vol.95, no.1-4, pp. 157-169, 2018.

[8] X. Zhao, C. Yao, Z. Zhao, et al. "Performance evaluation of online transformer internal fault detection based on transient overvoltage signals." IEEE Transactions on Dielectrics and Electrical Insulation, vol.24, no.6, pp. 39063915, 2017.

[9] B. Chen, W. J. Yuan, et al. "Recognition of highvoltage cable partial discharge signal based on adaptive fuzzy c-means clustering." International Journal of Pattern Recognition and Artificial Intelligence, vol.31, no.6, pp. 1759009, 2017,.

[10] J. Xiong, Y. Wang, G. M. Ma, et al. "Field applications of ultra-high frequency techniques for defect detection in GIS." Sensors, vol.18, no.8, pp. 2425, 2018.

[11] J. B. Ali, L. Saidi, S. Harrath, et al. "Online automatic diagnosis of wind turbine bearings progressive degradations under real experimental conditions based on unsupervised machine learning." Applied Acoustics, vol.132 pp. 167-181, 2018.

[12] V. Nasir, J. Cool, F. Sassani. "Acoustic emission monitoring of sawing process: artificial intelligence approach for optimal sensory characteristics selection." The International Journal of Advanced Manufacturing Technology, vol.102, no.9-12, pp. 4179-4197, 2019.

[13] X. Duan, T. Zhao, T. Li, et al. "Method for diagnosis of on-load tap changer based on wavelet theory and support vector machine." The Journal of Engineering, vol.2017, no.13, pp. 2193-2197, 2017.

[14] 0. M. Vasilevskyi, P. I. Kulakov, K. V. Ovchynnykov, et al. "Evaluation of dynamic measurement uncertainty in the time domain in the application to high speed rotating machinery." International Journal of Metrology and Quality Engineering, vol.8, pp. 25, 2017. 